Argumentation et Analyse du Discours

\title{
Bibliographie annotée des œuvres publiées de Michael Leff
}

\section{Steven R. Edscorn}

Traducteur : Sivan Cohen-Wiesenfeld

\section{(2) OpenEdition}

\section{Journals}

\section{Édition électronique}

URL : http://journals.openedition.org/aad/1076

DOI : 10.4000/aad.1076

ISSN : 1565-8961

\section{Éditeur}

Université de Tel-Aviv

Référence électronique

Steven R. Edscorn, « Bibliographie annotée des œuvres publiées de Michael Leff », Argumentation et Analyse du Discours [En ligne], 6 | 2011, mis en ligne le 15 avril 2011, consulté le 23 septembre 2019. URL : http://journals.openedition.org/aad/1076 ; DOI : 10.4000/aad.1076

Ce document a été généré automatiquement le 23 septembre 2019.

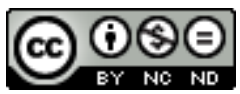

Argumentation \& analyse du discours est mis à disposition selon les termes de la licence Creative Commons Attribution - Pas d'Utilisation Commerciale - Pas de Modification 4.0 International. 


\title{
Bibliographie annotée des œuvres publiées de Michael Leff
}

\author{
Steven R. Edscorn \\ Traduction : Sivan Cohen-Wiesenfeld
}

\section{Livres}

1 Leff, Michael \& Fred Kauffeld (eds). 1989. Texts in Contexts: Dialogues on Significant Episodes in American Political Rhetoric [Textes en contexte: dialogues sur les épisodes significatifs de la rhétorique politique américaine] (Davis: Hermagoras Press)

Ce recueil de dialogues tente de recréer l'esprit et l'orientation des discussions tenues au cours du "Symposium du Wisconsin sur le discours public: études de cas de rhétorique politique ».

2 Horner, Winifred \& Michael Leff (eds). 1995. Rhetoric and Pedagogy: Their History, Philosophy, and Practice. Essays in Honor of James J. Murphy [Rhétorique et pédagogie : leur histoire, philosophie et pratique. Essais en l'honneur de James J. Murphy] (Mahwah, NJ: Lawrence Erlbaum)

Ces essais traitent de la pédagogie dans le domaine de la rhétorique à partir de l'époque classique, en passant par la Renaissance. Le dernier essai porte sur le thème « déconstruction et rhétorique ».

3 Andres, James, Michael Leff \& Robert Terrill. 1998. Reading Rhetorical Texts: An Introduction to Criticism [Lire les textes rhétoriques: une introduction à la critique] (Boston: Houghton Mifflin)

Ce manuel d'études secondaires présente s'une façon éclairante les principes de base de la critique rhétorique sur le plan théorique, et les illustre par des analyses des grands textes de l'art oratoire américain empruntées à divers spécialistes.

4 Leff, Michael. 1983. Rhetorical Timing in Lincoln's 'House Divided' Speech [Le moment propice rhétorique dans le discours de la "Maison divisée » de Lincoln] (Evanston, IL: Northwestern U.) 
Leff fait ici une analyse d'un discours de Lincoln, précédemment étudié par les historiens et les politologues, mais pas par les rhétoriciens. En portant une attention particulière aux mouvements temporels et spatiaux, aux métaphores et aux images, et en les reliant au contexte politique, il fournit un exemple de ce que la critique rhétorique peut apporter à la recherche universitaire et à la culture dans leur ensemble.

\section{Articles et essais}

5 Leff, Michael. 1971. "Reinterpreting Plato's Phaedrus" ["Réinterpréter Phèdre de Platon], Quarterly Journal of Speech 57, 334-347

L'auteur réfute l'argument de Brownstein selon lequel Platon ne considère jamais la rhétorique comme un art légitime, et selon lequel Phèdre représenterait une tentative "d'exiler la rhétorique à Coucou-les-Nuées » ${ }^{1}$. Pour Leff, cette position " dénature la philosophie de Platon» et «ignore ou simplifie à outrance des preuves importantes externes au texte ».

6 Leff, Michael. 1973. "The Latin Stylistic Rhetorics of Antiquity " «La rhétorique stylistique latine de l'Antiquité »], Speech Monographs 40, 273-279

Leff examine plusieurs manuels de figures latins, dont le Schemata Dianoeas et Lexeos ex Graecis Gorgiae Versa de Rutilius Lupus, De Figuris Sententiarum et Elocutionis d'Aquila Romanus, et De Figuris Sententiarum et Elocutionis de Julius Rufianius, afin d'élargir la conception prépondérante des les évolutions stylistiques, qu'il attribue à Wilbur Howell. Il suggère que le Liber de Schematibus et Tropis de Venerable Bede (701 apr. J.-C.), bien qu'il présente une perspective fondamentalement médiévale, dérive dans une grande mesure de textes latins plus anciens. Ceci admis, «on pourrait considérer la rhétorique des figures de l'âge helléniste jusqu'au dix-septième siècle comme faisant partie d'une tradition unique, bien que changeante ».

7 Leff, Michael C. 1973. "Redemptive Identification: Cicero's Catilinarian Orations » Mohrmann G. P., C. Stewart \& D. Ochs (eds). Explorations in Rhetorical Criticism ["L'identification rédemptrice: les Catilinaires de Cicéron", Explorations en critique rhétorique], (University Park, PA: Pennsylvania State University Press)

L'auteur avance que l'usage fréquent de l'hyperbole contre Catilina par Cicéron dans les Catilinaires suggère un rituel $d u$ bouc émissaire que Kenneth Burke appelle « identification rédemptrice ».

8 Leff, Michael \& G. P. Mohrmann. 1974. « Lincoln at Cooper Union: A Rhetorical Analysis of the Text » [« Lincoln à Cooper Union : une analyse rhétorique du texte »], Quarterly Journal of Speech 60, 346-358 ${ }^{2}$

L'un des travaux les plus importants de M. Leff. Leff et Mohrmann présentent une microanalyse (close reading) du discours électoral de Lincoln à Cooper Union. En portant leur attention sur les attentes qui suscite ce genre de discours électoraux, ainsi que sur le contexte de la campagne, Leff et Mohrmann prennent le contre-pied de l'opinion habituelle selon laquelle Lincoln tentait de se concilier l'auditoire du Sud. Ils suggèrent au contraire qu'il mettait en œuvre une décision stratégique visant à consolider l'investiture républicaine dans les Etats du Nord. 
Mohrmann, G. P. \& Michael C. Leff. 1974. «Lincoln at Cooper-Union: A Rationale for Neo-Classical Criticism» [«Lincoln à Cooper Union: le fondement rationnel de la critique néo-classique], Quarterly Journal of Speech 60, 459-4673

Mohrmann et Leff développent l'approche méthodologique utilisée dans "Lincoln à Cooper Union : une analyse rhétorique du texte». Ils proposent «de s'en rapporter à une théorie des genres afin d'introduire une analyse formelle dans la critique néoclassique " Cette démarche permet de résoudre un dilemme de la critique néoaristotélicienne issu de la distinction établie par Wicheln entre rhétorique et littérature - où les valeurs stables relèvent de la littérature, et le particulier de la rhétorique distinction qui limite l'évaluation des normes de la critique rhétorique à l'effet persuasif.

10 Leff, Michael. 1976. "St. Augustine and Martianus Capella: Continuity and Change in Fifth Century Latin Rhetorical Theory»["Saint Augustin et Martianus Capella: continuité et changement dans la théorie rhétorique latine au cinquième siècle »], Communication Quarterly 24, 2-9

Leff affirme que, malgré des différences évidentes, Augustin et Martianus partageaient une conception cicéronienne du lien entre la sagesse et l'éloquence. Tous deux tentèrent de préserver ce rapport dans une culture qui valorisait le contemplatif et l'absolu par rapport au pragmatique et au relatif. Augustin remplaça les orateurs païens par l'Ecriture sainte comme source première de l'éloquence. Martianus tenta de sauvegarder cette idée dans un manuel technique présentant le sens spirituel à travers l'allégorie. L'étude de Leff suggère que les traditions rhétoriques s'adaptent à l'évolution des cultures.

11 Leff, Michael. 1978. "The Logician's Rhetoric: Boethius' De Differentiis Topicis, Book IV ", Murphy, James J. (ed.). Medieval Eloquence: Studies in the Theory and Practice of Medieval Rhetoric ["La rhétorique du logicien: De Differentiis Topicis de Boèce, Livre IV", L'éloquence médiévale: études de la théorie et de la pratique de la rhétorique médiévale] (Berkeley, CA: University of California Press)

Leff montre que Boèce incorpore la rhétorique à la dialectique en la réduisant à des catégories propositionnelles abstraites. Ceci entraine une rupture plus complète avec la tradition classique que chez d'autres auteurs médiévaux du début du Moyen-âge, qui se sont contentés d'adapter les paradigmes classiques aux circonstances de leur époque. Leff se différencie des positions de Boèce par rapport à Aristote et aux humanistes de la Renaissance, et conclut que ces différences «mettent l'accent sur des divergences fondamentales sur la nature et le but du discours humain ».

12 Leff, Michael. 1978. "In Search of Adriane's Thread: A Review of the Recent Literature on Rhetorical Theory. » ["A la recherche du fil d'Ariane : revue de la littérature récente sur la théorie rhétorique »] Central States Speech Journal 29, 73-91

Dans l'un de ses travaux les plus importants, Leff affirme que l'un des fils conducteurs commun de la théorie rhétorique contemporaine est l'intérêt manifesté envers le lien qui unit la rhétorique et l'épistémologie, un intérêt qui marque une rupture significative par rapport à la théorie rhétorique antérieure. Il explore les diverses manières dont la rhétorique peut être considérée comme épistémologique, ou l'épistémologie comprise comme une rhétorique. Il remarque que les travaux sur la communication verbale souffrent $d u$ fait que ces concepts ne sont pas reliés à l'expérience pratique : les travaux théoriques restent distincts des études textuelles. 
13 rhétorique : l'état de l'art »], Western Journal of Speech Communication 44, 337-349

Faisant le compte rendu des articles d'un numéro spécial de la revue et les faisant dialoguer avec ceux d'un numéro similaire de 1957, Leff conclut que la critique rhétorique en tant que discipline a réussi à développer un nombre important de théories, et que son expansion au-delà du néo-aristotélisme a eu des effets salutaires. Cependant, deux problèmes subsistent : une tendance à construire une théorie séparée de la pratique rhétorique, et une tendance à appliquer des théories sur les textes rhétoriques d'une façon mécanique. Leff est particulièrement impressionné par l'essai d'Edwin Black qui propose de passer d'une critique fondée sur la théorie à une critique basée sur le texte, mais il remarque que la distinction de Black est trop catégorique. Il suggère que la critique doit «faire résonner » le texte par rapport à la fois à la théorie et à l'expérience subjective.

Leff, Michael. 1981. «Forms of Reality in Plato's Phaedra " [ Les formes de la réalité dans Phèdre de Platon »], Rhetoric Society Quarterly 11, 21-23

Leff suggère que la position épistémologique de Platon dans Phèdre rend compte d'un véritable paradoxe rhétorique parce qu'il doit exprimer l'ineffable à travers le médium corporel du langage.

Leff, Michael C. \& Dean E. Hewes. 1981. «Topical Invention and Group Communication: Towards a Sociology of Inference ", Dimensions of Argument: Proceedings of the Second Summer Conference on Argumentation ["L'invention topique et la communication de groupe : vers une sociologie de l'inférence ", Les dimensions de l'argument: actes de la seconde conférence d'été sur l'argumentation], (Annandale, VA: SCA), 770-789

Leff et Hewes tentent de trouver un point de jonction entre l'invention rhétorique et la communication de groupe, en particulier à l'intérieur de petits groupes ayant pour but la prise de décision, à la lumière des Topiques d'Aristote. Ils concluent que l'argumentation rhétorique est « à la fois un outil pour l'accomplissement des décisions de groupe et un produit de ce processus de prise de décision ». Ils suggèrent d'approfondir cette recherche afin de fournir un cadre plus large à ces observations isolées.

Leff, Michael C. 1982. "Concrete Abstractions: A Response to Skopec and Anderson » [«Les abstractions concrètes : réponse à Skopec et Anderson»], Pennsylvania Speech Annual 38, 21-24

Leff répond à deux interprétations divergentes de Douglas Ehninger : l'une s'accordant avec son refus de la théorie classique (Skopec), et l'autre mettant l'accent sur les topoï classiques (Anderson). Leff est de l'avis d'Anderson et développe son argument. Il fournit des exemples d'auteurs comme Richard Weaver, Kenneth Burke, Chaïm Perleman et Wayne Booth qui considèrent la théorie des topoï comme valable dans la mesure où elle tient compte du contexte. Leff relie sa propre argumentation à une tradition autoréflexive et humaniste partant d'Isocrate et de Cicéron pour aller jusqu'à Booth et d'autres, afin de montrer la continuité de la tradition rhétorique.

Leff, Michael. 1983. "Topical Invention and Metaphoric Interaction" [ Invention topique et interaction métaphorique»], Southern Speech Communication Journal 48, 214-228

Leff avance que la distinction communément admise entre les topiques et les tropes comme étant, respectivement, synchroniques et diachroniques, disparaît à un certain 
degré dans les textes rhétoriques réels. Il montre, par des exemples, comment les structures métaphoriques s'insèrent dans le développement des arguments propositionnels, et fusionnent avec eux.

18 Leff, Michael. 1983. "The Lore of Topical Invention: From Cicero to Boethius » [« La science de l'invention topique : de Cicéron à Boèce »], Rhetorica 1, 23-44

En dépit de son titre limitatif, ce travail retrace en fait l'évolution de la conception des topiques d'Aristote et d'Hermagoras à Boèce, en passant par Cicéron, Quintilien et d'autres auteurs latins plus tardifs. Leff remonte ainsi à la source d'une dichotomie contemporaine entre une tradition aristotélicienne qui permet plus de cohérence, et une tradition cicéronienne qui résiste au processus de division entre forme et contenu, et maintient davantage le lien des topiques avec les situations réelles.

19 Browne, Steve \& Michael Leff. 1985. « Rhetoric and Political Judgment: Edmund Burke's Paradigm ", Argument and Social Practice: Proceedings of the Fourth SCA/AFA Conference on Argumentation [ "Rhétorique et jugement politique : le paradigme d'Edmund Burke», Argument et pratique sociale: Actes de la quatrième Conférence SCA/AFA sur l'argumentation] (Annandale, VA: SCA), 193-210

Browne et Leff mènent une analyse serrée du «Discours aux électeurs de Bristol » d'Edmund Burke, montrant que celui-ci développe une théorie du jugement basé sur une situation politique particulière, et matérialise cette théorie par son discours.

20 Leff, Michael. 1985. "Recovering Aristotle: Rhetoric Politics, and the Limits of Rationality»["Redécouvrir Aristote: la politique rhétorique et les limites de la rationalité »], Quarterly Journal of Speech 71, 262-272

Leff passe en revue deux livres de politologues portant sur la Rhétorique d'Aristote: Aristote et le raisonnement politique: un commentaire de La Rhétorique d'Arnhart et Le jugement politique de Beiner, dans le but d'acquérir sur l'ouvrage d'Aristotedes perspectives issues de chercheurs qui restent étrangers à la dichotomie entre rhétoriciens aristotéliciens et postmodernes. Les deux auteurs tentent de défendre la «rationalité » de la Rhétorique d'Aristote. Leff fait de sérieuses critiques à ces deux livres, reconnaissant cependant la valeur du travail de Beiner, suffisamment original et convaincant pour inviter à une reconsidération de la relation entre la Rhétorique d'Aristote et le tournant postmoderne de la rhétorique.

21 Leff, Michael \& Margaret Organ Procario. 1985. "Rhetorical Theory in the Twentieth Century ", Benson, Thomas (ed.). Speech Communication in the Twentieth Century [« La théorie rhétorique au vingtième siècle ", Benson, Thomas (éd.). La communication verbale au vingtième siècle], (Carbondale, IL: Southern Illinois University Press), 3-27

Leff et Procario passent en revue l'évolution de la théorie rhétorique, dans le cadre des études de communication. Ils concluent que la théorie rhétorique a beaucoup progressé pendant les cinquante années précédant la publication de l'article, mais que peu d'innovations marquantes sont venues de l'intérieur même de la discipline.

22 Leff, Michael. 1986. "Genre and Paradigm in Book II of De Oratore " ["Genre et paradigme dans le Livre II de l'Art oratoire »], Southern Journal of Speech Communication 51, 308-325

Leff affirme que Cicéron met en lumière la tension entre le paradigme isocratien et le genre aristotélicien dans le Livre II de l'Art oratoire. Cicéron adopte tout d'abord le parti d'Isocrate, optant pour une conception plus extensive de la rhétorique basée sur la pratique d'un art guidée par des préceptes. Cependant, il considère qu'il est bon 
d'utiliser les genres selon Aristote d'une manière plus limitée, pour « organiser des éléments de compréhension fournis par l'étude de cas particuliers ». Leff recommande l'approche ironique de Cicéron à l'égard des tensions entre ces perspectives pour les futures études de théorie rhétorique.

Leff, Michael. 1986. «Textual Criticism: The Legacy of G. P. Mohrmann » [« La critique textuelle : l'héritage de G. P. Mohrmann »], Southern Journal of Speech Communication 51, 337-389

Dans un travail en l'honneur du défunt G.P. Mohrmann, Leff illustre l'approche de ce dernier sur la critique textuelle en commentant son analyse du "Discours sur la réception des pétitions sur l'Abolition » de Calhoun et le discours de Lincoln à Cooper Union. Entre autres, Leff discute la manière dont Mohrmann approche les textes à travers l'étude des métaphores spatiales et temporelles, du jugement et de l'ostracisme rituel.

Leff, Michael. 1987. "The Habitation of Rhetoric ", Wenzel, Joe (ed.), Argument and Critical Practice: Proceedings of the Fifth AFA/SCA Conference on Argumentation [" Le cadre de la rhétorique ", Wenzel, Joe (ed.). Argumentation et pratique critique: Actes de la cinquième conférence AFA/SCA sur l'argumentation] (SCA: Annandale, VA), 1-8

Leff propose le concept de décorum ${ }^{4}$ considéré comme la réunion de l'esthétique et de l'argumentatif dans chaque œuvre rhétorique particulière, comme un moyen de faire le lien entre le processus rhétorique et son résultat.

Leff, Michael. 1987. «Modern Sophistic and the Unity of Rhetoric », Nelson, J., A. Megill, and D. McClosky (eds). The Rhetoric of the Human Sciences: Language and Argument in Public Affairs [" La sophistique moderne et l'unité de la rhétorique », Nelson, J., A. Megill, \& D. McClosky (éds). La rhétorique des sciences humaines: langage et argumentation dans les affaires publiques] (Madison: U. of Wisconsin Press), 19-37

Leff affirme que le discours des sciences humaines et celui des affaires publiques doivent tous deux être considérés comme rhétoriques. Cependant, il suggère qu'il s'agit de groupes d'activité distincts dans le domaine de la rhétorique. Cette distinction dérive des attentes et des capacités de l'auditoire, mais elle affecte la rhétorique au sens large en termes de langage, de preuves, de style, d'argument etc. Il recommande une approche "sophistique » qui évalue le discours des sciences humaines à la lumière de ses conditions propres.

Leff, Michael. 1988. «Dimensions of Temporality in Lincoln's Second Inaugural » [« Les dimensions de la temporalité dans le deuxième discours inaugural de Lincoln »], Communication Reports 1, 26.31

Leff fait une analyse minutieuse du Deuxième discours inaugural de Lincoln montrant que le mouvement temporel «encadre l'action des divers éléments argumentatifs et stylistiques, les imbriquent dans un champ unifié d'action textuelle, et projette ce champ sur les évènements publics qui forment le sujet du discours ». Il affirme que les analyses antérieures n'ont pas saisi ce point parce qu'elles n'ont pas pris en compte l'ensemble du texte.

Leff, Michael. 1988. « Serious Comedy: The Strange Case of Dr. Vitanza » [« La comédie sérieuse : l'étrange cas de Vitanza »], Rhetoric Review 6, 237-245

Leff reproche à Vitanza de présenter une vue totalement binaire de l'histoire de la rhétorique, séparant deux traditions, l'une rationnelle et "disciplinariste", incluant, entre autres, aussi bien Cicéron et Perelman que Platon et Pierre de la Ramée ; l'autre 
post-moderne, "comique et sophistique». Vitanza propose comme solution une "rhétorique métadisciplinaire", combinant ces deux traditions. Leff affirme que ces deux branches ont déjà été entrelacées, sur des modes complexes et ironiques que Vitanza a négligés, comme il n'a pas pris en compte la métadisciplie rhétorique qu'offrent, entre autres, Isocrate, Cicéron et Quintilien.

Leff, Michael. 1989. «Burke's Ciceronianism », Simmons, Herbert \& Trevor Melia (eds). The Legacy of Kenneth Burke ["Le cicéronianisme de Burke», Herbert \& Trevor Melia (éds). L'héritage de Kenneth Burke], (Madison: U. of Wisconsin Press), 115-127

Leff met face à face les "Quatre maîtres tropes » de Kenneth Burke De l'Art oratoire de Cicéron afin de mettre en lumière leurs similitudes concernant la question de la réalisation textuelle et de l'ironie. Ces ressemblances dériveraient du fait que Burke et Cicéron partagent la même philosophie du « langage comme action ».

Rosteck, Tom \& Michael Leff. 1989. «Piety and Propriety: An Interpretation and Application of Key Terms in Kenneth Burke's Permanence and Change " ["Piété et décence : interprétation et application de termes clés dans Permanence et changement de Kenneth Burke »], Western Journal of Speech, 53, 327-341

Rosteck et Leff affirment que le concept de piété chez Kenneth Burke inclut la notion plus ancienne de "décence », " correction », centrale pour Cicéron et d'autres auteurs classiques. Analysant un texte rhétorique de l'anarchiste Voltairine de Cleyre, ils montrent comment cette dynamique autorise une critique conjointe du style et de l'argument, approche qui permet à son tour l'étude de textes particuliers.

Leff, Michael. 1990. "Decorum and Rhetorical Interpretation: The Latin Humanistic Tradition and Contemporary Rhetorical Theory» [ "Decorum et interprétation rhétorique : la tradition humaniste latine et la théorie rhétorique contemporaine »], Vichiana 3e série, 107-126

Leff soulève le problème, posé par Ricœur et Todorov, selon lequel «la rhétorique semble incapable de contenir les pulsions contraires» du formalisme et du fonctionnalisme. Il trouve une solution dans Art oratoire et dans l'Orateur, dans lesquels Cicéron traite du décorum 5 . Pour Leff, le decorum permet d'opérer la médiation entre la forme et le fond du discours.

31 Leff, Michael. 1990. "Prudential Argument and the Use of History in FDR's 'Commonwealth Club' Address", Proceedings of the $2^{\text {nd }}$ International Conference on Argumentation, vol. $1 \mathrm{~b}$ [«L'argument de prudence et l'utilisation de l'histoire dans le Discours au Club du Commonwealth de Franklin D. Roosevelt ", Actes de la 2e conférence internationale sur l'argumentation] (Amsterdam: Sic Sat), 931-936

Leff montre que le «Discours au Club du Commonwealth » de Roosevelt constitue « un champ intense d'action rhétorique qui aligne des intérêts politiques en situation avec des thèmes historiques et économiques plus larges ». S'inspirant de Robert Cox, Leff suggère que cette démarche constitue une forme d'argument d'utilité susceptible de surmonter le dualisme entre l'innovation et l'histoire inhérent à la culture américaine, lui-même générateur de dilemmes au sein du discours politique.

Leff, Michael \& Andrew Sachs. 1990. «Words the Most Like Things: Iconicity and the Rhetorical Text»[ «Des mots au plus près des choses: l'iconicité et le texte rhétorique »], Western Journal of Speech Communication 54, 252-273

Montrant que le style est, à chaque niveau, inséparable du contenu dans le « Discours aux électeurs de Bristol » d'Edmund Burke, Leff et Sachs affirment que l'approche de la 
microanalyse (close reading) peut véritablement mettre le doigt sur l'idéologie qui s'inscrit dans la texture du discours. Ceci fait du texte une «icône » de l'idéologie à l'intérieur de formations discursives plus larges.

Leff, Michael. 1992. «Teaching Public Speaking as Composition » [L'enseignement du discours public comme composition »], Basic Course Annual 4,116-122

Leff affirme qu'en mettant l'accent sur l'enseignement de la composition du discours public, les rhétoriciens des départements de communication pourraient redonner vigueur aux cours sur le discours public en combinant théorie et pratique.

Leff, Michael. 1992. «Things Made by Words: Reflections on Textual Criticism » [Les choses faites par les mots : réflexions sur la critique textuelle], Quarterly Journal of Speech 78, 223-231

Prenant pour exemples le « Discours à l'Exposition d'Atlanta » de Booker T. Washington et «Sur M. Booker T. Washington et d'autres" de W. E. B. DuBois, Leff prône la controverse directe comme l'une des conditions permettant à la microanalyse (close reading) de dépasser le texte en tant qu'unité spécifique.

Leff, Michael. 1993. "The Uses of Aristotle's Rhetoric in Contemporary American Scholarship " [ "Les usages de la rhétorique d'Aristote dans la recherche américaine contemporaine »], Argumentation 7, 313-327

Leff examine la littérature secondaire sur la Rhétorique d'Aristote, et conclut que ses perspectives produisent une «double distorsion». D'une part, la Rhétorique est "resserrée au point de représenter un point de vue unique et dénué de complexité ", de l'autre elle est « dilatée au point de bloquer la perception de quoi que ce soit d'autre chose dans l'histoire de la rhétorique ».

Leff, Michael. 1994. «Recherches américaines sur les lieux», Plantin, Christian (éd.), Lieux communs, topoi, stéréotypes, clichés (Paris : Kimé), 506-5176

Analyse des recherches américaines récentes sur l'invention topique, replacées dans le cadre plus large de l'évolution de la conception de la rhétorique. Longtemps rejetés comme résidus obsolètes du système classique, les lieux ont été réhabilités à partir des années 70, mais ce sont surtout les "néo-humanistes» qui, dans leur tentative d'étendre le domaine de la rhétorique en redonnant vigueur à la tradition humaniste, ont placé l'invention topique au cœur même de la rhétorique. Dans cette perspective, les lieux, loin d'être considérés comme des types statiques d'arguments, deviennent des stratégies mobiles, dérivées d'un usage antérieur, mais capables de se réincarner dans des cas nouveaux, permettant au sujet d'exercer une pensée individuelle tout en s'adaptant «avec souplesse et précision aux exigences de la communauté ». Mettant l'accent sur la pratique effective plus que sur l'abstraction théorique, ils ont pour fonction de nourrir le processus du débat antithétique au centre de la méthode humaniste.

Leff, Michael. 1994. "Cicero's Redemptive Identification: Commentary », Nothstine, W., C. Blair, \& G. Copeland (eds.). Critical Questions: Invention, Creativity, and the Criticism of Discourse and Media ["L'identification rédemptrice de Cicéron: un commentaire", Nothstine, W., C. Blair, \& G. Copeland (éds.). Questions de critique : Invention, créativité et la critique du discours et des média] (New York: St. Martins), 323-326

Leff réexamine son article de 1973 "Identification rédemptrice: les Catilinaires de Cicéron ". Il suggère que, bien que l'article ne manquât pas de mérite, ses premiers travaux reflètent une tendance à utiliser les théories en fonction des besoins de la 
cause et à multiplier les théories à l'intérieur du champ. Il émet l'hypothèse selon laquelle son "jeune moi » avait encore besoin de déterminer clairement son objectif dans le domaine de la critique.

Terrill, Robert \& Michael Leff. 1995. «The Polemicist as Artist: W. E. B. Dubois 'On Mr. Booker T. Washington and Others' ", Jackson, Sally \& al. (Eds.) Argumentation and Values: Proceedings of the $9^{\text {th }}$ AFA/SCA Conference on Argumentation [" Le polémiste comme artiste : W. E. B. Dubois "M. Booker T. Washington et autres" ", Jackson, Sally \& al. (éds). Argumentation et valeurs: Actes de la $9^{e}$ Conférence AFA/SCA sur l'argumentation ] (Annandale, VA: SCA), 230-236

Terrill et Leff définissent l'argumentation dans l'essai de Dubois comme « ancrée dans le mouvement rhétorique plus large du texte - intimement liée à la voix que Dubois projette, elle en est inséparable. Ils concluent que Dubois « trouve une voix critique qui contourne l'hégémonie silencieuse de [Booker T.] Washington et la défie » et que cette voix offre également une "alternative au style et à la substance du leadership de [Washington]».

Zulick, Meg \& Michael Leff. 1995. "Time and True Light in Lucretia Coffin Mott's Discourse on Woman. » [ « Le temps et la vraie lumière dans le Discours sur la femme de Lucretia Coffin Mott »], Rhetoric Society Quarterly 25, 20-31

Zulick et Leff approfondissent une précédente analyse de Karlyn Kohrs Campbell du « Discours sur la femme » en en inversant le point focal. Au lieu d'examiner l'identité de Quaker de L. Mott à la lumière de sa rhétorique féministe, ils étudient sa rhétorique féministe au prisme de son identité de Quaker «Hicksite " ${ }^{7}$. Le résultat est que «le poids relatif du dit se déplace » en "éclairant les relations textuelles entre le locuteur et l'auditoire », et en permettant également de faire apparaître les stratégies inventives de Mott.

Leff, Michael. 1996. "Commonplaces and Argumentation in Cicero and Quintilian" [«Lieux communs et argumentation chez Cicéron et Quintilien], Argumentation 10, $445-452$

Leff s'inspire de Cicéron et de Quintilien pour affirmer que les lieux communs méritent une plus sérieuse attention en rhétorique. A Cicéron, Leff emprunte l'idée selon laquelle les lieux communs ne sont pas des arguments, mais « des produits finis qui intègrent l'argument logique, l'appel émotionnel et le style dans une structure unique ». Chez Quintilien, Leff retrouve une distinction entre « un lieu commun stérile, purement verbal, et un autre, heuristique, qui aide l'orateur à agencer la matière en une forme appropriée à une sorte de tâche spécifique ».

41 Leff, Michael. 1996. «Agency, Performance, and Interpretation in Thucydides' Account of the Mitylene Debate ", Johnsonte, Christopher (ed.). Theory, Text, Context: Issues in Greek Rhetoric and Oratory ["Agentivité, représentation et interprétations dans le compte-rendu par Thucydide du débat de Mitylene », Johnsonte, Christopher (éd.). Théorie, Texte, Contexte: questions dans la rhétorique et l'art oratoire grecs] (Albany: SUNY Press), 97-106

Leff critique l'affirmation de Gaonkar selon laquelle la rhétorique classique, en raison, selon lui, de son "idéologie de l'agentivité humaine", ne traitait pas des questions d'interprétation ni d'analyse culturelle. Leff examine un segment de l'Histoire de Thucydide, et conclut que, si celui-ci souscrit effectivement à cette idéologie, il présente cependant cette agentivité comme en décadence dans l'Athènes post- 
péricléenne, car les agents « ont échoué à rendre compte des contraintes qui pèsent sur leurs actions ». Thucydide utilise donc l'idéologie pour critiquer la culture.

Leff, Michael. 1996. "The Idea of Rhetoric as Interpretive Practice: A Humanist's Response to Gaonkar ", Gross, Alan and William Keith (eds). Rhetorical Hermeneutics: Invention and Interpretation in the Age of Science [" L'idée de la rhétorique comme pratique interprétative : une réponse humaniste à Gaonkar ", Gross, Alan \& William Keith (éds). Herméneutique rhétorique: invention et interprétation à l'âge de la science] (Albany: SUNY Press), 89-101

Leff développe l'argument avancé dans « Agentivité, représentation et interprétation dans le compte rendu par Thucydide du débat de Mitylene » (voir ci-dessus). Il réfute de nouveau l'affirmation de Gaonkar selon laquelle «l'idéologie de l'agentivité humaine » propre à la rhétorique classique différencie radicalement la rhétorique humaniste du «tournant interprétatif» de la rhétorique post-moderne. Cette fois-ci, Leff accuse Gaonkar de laisser cette "rigidité binaire» orienter sa lecture des travaux des chercheurs humanistes dans une direction qui renforce sa propre théorie. Pour étayer ce point, Leff examine la critique par Gaonkar du travail de John Angus Campbell sur Charles Darwin.

Leff, Michael. 1997. "Hermeneutical Rhetoric ", Jost, Walter \& Michael Hyde (eds). Rhetoric and Hermeneutics in our Time ["Rhétorique herméneutique", Jost, Walter \& Michael Hyde (éds). Rhétorique et herméneutique à notre époque] (New Haven: Yale University Press), 196-214

Leff tente d'établir un lien, à l'intérieur de la discipline de la critique rhétorique, entre les praticiens de la critique textuelle, comme lui-même, qui cherchent à interpréter des textes spécifiques et tendent vers le réalisme textuel, et les partisans de la critique rhétorique, qui mettent l'accent sur la réception par l'auditoire et tendent vers un «idéalisme de la lecture ». Il répond ici aux partisans de la critique rhétorique qui suggèrent que les deux orientations sont incompatibles. Leff remarque que le texte et l'auditoire concourent à déterminer le sens. Pour lui, tant les tenants de la critique textuelle que ceux de la critique rhétorique doivent offrir leur contribution à la vaste communauté des critiques rhétoriques. Cela signifie qu'un partisan de la critique textuelle doit s'engager dans une certaine mesure dans une interprétation approfondie et contextuelle des textes, et qu'une critique rhétorique doit accorder une certaine attention aux textes concrets.

Leff, Michael. 1998. "Lincoln Among the Nineteenth-Century Orators", Benson, Thomas (ed.). Rhetoric and Political Culture in Nineteenth-Century America [« Lincoln parmi les orateurs du dix-neuvième siècle ", Benson, Thomas (éd.). Culture politique et rhétorique dans l'Amérique du dix-neuvième siècle)] (East Lansing: Michigan State University Press), 131-156

Leff étudie la façon dont trois discours du dix-neuvième siècle: "Le nouveau Sud " d'Henry Grady, le «Discours à la mémoire de Lincoln au Mémorial Freedman » de Frederick Douglas et « un Lear moderne » de Jane Adams se sont appropriés la parole de Lincoln. Il note que ces trois orateurs l'utilisent pour relier les innovations à un contexte historique. Il affirme de plus que, en dépit des conceptions et de l'apport des approches post-modernes et idéologiques de la rhétorique, des études consacrées à des discours entiers sont nécessaires pour «sonder la complexité des processus interprétatifs et productifs qui participent à l'acte rhétorique ». 
Michael. 1998. "Cicero's Pro Murena and the Strong Case for Rhetoric " [Pro Murena de Cicéron comme le cas fort de la rhétorique »], Rhetoric and Public Affairs 1, 61-88

Leff affirme que Pro Murena " peut être lu comme l'incarnation d'un type de jugement politique spécifiquement lié à la prudence, au decorum et à l'action et dans le cadre duquel les compétences rhétoriques ne sont pas considérées uniquement comme des outils de persuasion mais comme un équipement pour bien mener sa vie». Ceci complexifie le modèle du processus persuasif permettant d'appréhender la rhétorique de Cicéron, de façon à circonvenir le problème soulevé par une conception instrumentale de la rhétorique qui éliminerait toute possibilité de concevoir l'orateur comme agent éthique.

Keith, William, Steve Fuller, Alan Gross \& Michael Leff. 1999. « Taking Up the Challenge: A Response to Simons » [«Relever le défi : une réponse à Simon »]. Quarterly Journal of Speech 85, 330-338

Keith, Fuller, Gross et Leff avancent que Gaonkar a soulevé trois points que la rhétorique en tant que discipline doit prendre au sérieux. Ils affirment que son expansion au-delà des questions politiques est irréversible, qu'elle a besoin de trouver une manière de définir ses limites, et que les rhétoriciens doivent être plus ou moins prompts à faire effectuer sans discrimination des emprunts à d'autres disciplines.

47 Leff, Michael. 2000. "Abraham Lincoln's Rhetorical Leadership " [ Le leadership rhétorique d'Abraham Lincoln »], Rhetoric and Public Affairs 3, 1

Introduction à un numéro spécial de Rhetoric and Public Affairs consacré à la rhétorique de Lincoln.

Leff, Michael \& Jean Goodwin. 2000. «Dialogic Figures and Dialectical Argument in Lincoln's Rhetoric » [ "Figures dialogiques et argument dialectique dans la rhétorique de Lincoln »], Rhetoric and Public Affairs 3, 59-69

Leff et Goodwin affirment que « Lincoln utilise continuellement et avec efficacité des figures dialectiques ou dialogiques comme la prolepse, la prosopopée et la correction. Ces figures l'aident à recadrer ses textes rhétoriques dans un univers argumentatif et lui offrent la possibilité de formuler et de dépasser des positions qui diffèrent des siennes. L'étude de cet aspect de la rhétorique de Lincoln contribue à expliquer le pouvoir de ses discours en même temps qu'elle permet de relier la pratique rhétorique à la recherche contemporaine en logique informelle ».

Leff, Michael \& Jean Goodwin. 2000. «Douglas Walton on Ad Hominem Argument » [«Douglas Walton sur l'argument Ad Hominem»], Argumentation and Advocacy 36, $177-178$

Leff et Goodwin introduisent un numéro d'Argumentation and Advocacy dans lequel Douglas Walton présente une approche de l'argument ad hominem permettant d'intégrer au domaine de la logique informelle des considérations sur le caractère; plusieurs étudiants de l'Université du Nord-Ouest appliquent cette conception aux débats du Congrès des Etats-Unis.

Leff, Michael. 2000. "Rhetoric and Dialectic in the Twenty-First Century " [«Rhétorique et dialectique au vingt-et-unième siècle »], Argumentation 14, 241-254

Leff présente « certaines différences entre la rhétorique et la dialectique telles qu'elles sont conçues dans la tradition pré-moderne, et qui offrent une base pour mieux 
comprendre les évolutions plus récentes ", en vue de caractériser des approches contemporaines de la dialectique. Il argumente en faveur d'une conception de la dialectique comme discipline hybride située entre la logique et la rhétorique ou, en d'autres termes, entre une rationalité exclusivement propositionnelle et une vision strictement instrumentale de la persuasion ».

51 Leff, Michael. 2000. «Rhetorical Disciplines and Rhetorical Disciplinarity: A Response to Mailloux » [ ¿Disciplines et disciplinarité rhétoriques: une réponse à Mailloux»], Rhetoric Society Quarterly 20, 83-93

Leff appuie l'appel de Mailloux à une coopération interdisciplinaire entre les rhétoriciens des départements d'Anglais et ceux des départements de Communication. Il affirme que les rhétoriciens du département de Communication ont une conception moins développée de leur discipline que leurs homologues en Anglais, en raison de l'influence prolongée des experts en sciences sociales et d'un lien plus faible avec la pédagogie dans les départements de Communication.

Leff, Michael. 2001. «Lincoln at Cooper Union: Neo-Classicism Revisited » [« Lincoln à Cooper Union : une approche néo-classique revue »], Western Journal of Communication $65,323-348$

En réponse à une critique de Jasinski, Leff ajoute une dimension intertextuelle à l'analyse du discours de Lincoln à Cooper Union qu'il avait réalisé avec Mohrmann en 1974. Il avance que la microanalyse (close reading) doit prêter attention non seulement à la situation concrète, mais également aux relations intertextuelles. Il suggère de même que les études intertextuelles ne doivent pas perdre la trace des situations concrètes. Enfin, il cite deux interprétations différentes d'un même passage, la sienne et celle de Jasinski, pour répondre à ceux qui prétendent que l'exercice de "close reading " n'autorise qu'une lecture unique.

Leff, Michael. 2002. "The Relation Between Dialectic and Rhetoric in a Classical and Modern Perspective ", van Eemeren \& P. Houtlosser (eds). Dialectic and Rhetoric: The Warp and Woof of Argumentative Analysis ["La relation entre dialectique et rhétorique selon les perspectives classique et moderne», van Eemeren \& P. Houtlosser (éds). Dialectique et rhétorique : le soubassement de l'analyse argumentative ] (Dordrecht: Kluwer), 53-64

Leff présente un bref historique de la relation entre la rhétorique et la dialectique, puis développe une argumentation similaire à celle qu'il avait exposé dans « Rhétorique et dialectique au vingt-et-unième siècle » (2000).

Leff, Michael. 2002. "Isocrates, Rhetoric, and the Idealization of Civic Education", Bourdouris, Konstantine \& John Poulakos (eds). The Philosophy of Communication, Vol. 2 (Athens: Ionia Publications) ["Isocrate, la rhétorique et l'idéalisation de l'éducation civique", Bourdouris, Konstantine \& John Poulakos (éds). La philosophie de la communication, vol. 2], 120-125

Leff soutient que les incohérences et ambiguïtés apparentes dans la rhétorique d'Isocrate n'indiquent pas qu'il manque d'idéalisme, mais dévoilent plutôt un idéal qui « requiert une adaptation aux circonstances changeantes du monde réel ».

Leff, Michael. 2003. "Tradition and Agency in Humanistic Rhetoric " [ Tradition et agentivité dans la rhétorique humaniste »], Philosophy and Rhetoric 36, 135-147

Utilisant comme texte l'Aeropagitica d'Isocrate, Leff plaide pour une approche plus dynamique de la tradition dans la rhétorique humaniste où elle «contraint l'art 
rhétorique tout en lui étant indispensable, limite les possibilités de prestation, tout en donnant à la prestation rhétorique une place signifiante dans l'histoire de la communauté ». Cette conception est à l'origine du sens ambigu de l'agentivité dans la rhétorique humaniste, où l'orateur « doit faire preuve de sa virtuosité individuelle en présentant et en la représentant la tradition, tout en affirmant sa solidarité avec l'auditoire ".

Leff, Michael. 2003. «Rhetoric and Dialectic in Martin Luther King's 'Letter From a Birmingham Jail' ", van Eemeren, F. H., J. A. Blair, C. A. Willard \& A. F. S. Henkemans (eds). Anyone Who Has a View: Theoretical Contributions to the Study of Argumentation (Dordrecht: Kluwer) [ «Rhétorique et dialectique dans la Lettre de la prison de Birmingham de Martin Luther King ", van Eemeren, F. H., J. A. Blair, C. A. Willard \& A. F. S. Henkemans (éds). Quiconque possédant un point de vue: contributions théoriques à l'étude de l'argumentation], 671-679

Leff examine l'argumentation de King dans la "Lettre de la prison de Birmingham », et soutient qu'elle participe de l'évocation rhétorique. Il suggère que les experts de logique informelle et les rhétoriciens ont beaucoup à apprendre les uns des autres.

Leff, Michael. 2003. « Fortolkende retorisk kritik », traduit par Lisa Villadsen, Rhetorica Scandinavica, 26, 6-20

Dans une microanalyse (close reading) de la lettre de Birmingham de Martin Luther King, Leff illustre les implications de la critique conceptuellement orientée, terme introduit par James Jasinski. Appelant sa propre démarche "une critique interprétative », il soutient que «la théorie» est une activité continue réalisée en lien étroit avec l'examen de la pratique rhétorique et que les constatations critiques sont horizontalement reliées entre elles par le moyen de l'analogie et de l'abduction, et non verticalement (du cas particulier aux principes). Les études critiques sont donc reliées conceptuellement, plutôt que méthodologiquement, à travers la réflexion sur la manière dont les principes et les concepts rhétoriques figurant dans un contexte particulier peuvent se matérialiser de façon différente dans un autre. Cette lecture du texte de King tourne autour des concepts de l'agentivité rhétorique, de la rhétorique " éthotique ", de la synecdoque et de l'ambiguïté stratégique, et analyse comment il fait face aux préoccupations à la fois des auditoires noirs et des blancs (Résumé de Lisa Villadsen).

Leff, Michal \& Ebony Utley. 2004. «Instrumental and constitutive Rhetoric in Martin Luther King's 'Letter From a Birmingham Jail' " [ Rhétorique instrumentale et constitutive dans la 'Lettre de la prison de Birmingham' de Martin Luther King], Rhetoric and Public Affairs 7, 37-52

Leff et Utley soutiennent que la «Lettre de la prison de Birmingham » présente une construction complexe du personnage de King, qui permet à celui-ci à la fois de critiquer le clergé blanc sans se l'aliéner, et de fournir à ses lecteurs noirs un modèle pour reconstruire leur propre sens de l'agentivité. Ceci suggère l'existence d'une relation souple entre l'ethos comme instrument de persuasion et l'ethos comme outil de construction de l'identité.

59 Leff, Michael.2004. "Isocrates, Tradition, and the Rhetorical Version of Civic Education », Poulakos, T. \& D. Depew (eds). Isocrates and Civic Education [« Isocrate, la tradition et la version rhétorique de l'éducation civique »] (Austin: U. of Texas Press), 235-254 
Leff propose une brève discussion de la réception d'Isocrate au siècle dernier, et fait une analyse critique des autres essais de ce volume. Il affirme que «les oppositions apparentes» entre Isocrate et Platon «disparaissent lorsqu'Isocrate compare l'excellence rhétorique et politique, l'intérêt particulier et civique et le style artistique avec la pensée élevée »; il aboutit ainsi à l'idée d'une sorte "d'organicisme» de la rhétorique d'Isocrate.

Lunsford, Andrea \& Michael Leff. 2004. « Afterwords: A Dialogue » [«Après les mots : un dialogue »], Rhetoric Society Quarterly 34, 55-68

Andrea Lunsford exprime l'espoir que la rhétorique puisse contribuer à un "mariage entre les objectifs grecs antiques et allemands modernes de l'université [pédagogique et production de connaissance] » et que la fusion des départements de Communication et d'Anglais constitue un pas important dans cette direction. Suivant un argument avancé par Jeffrey Walker et par d'autres, Leff suggère que la priorité doit être donnée soit à l'un soit à l'autre. Il affirme ensuite que « les sensibilités post-modernes proposent de nouveaux fondements permettant de considérer l'histoire de la rhétorique comme une tradition d'enseignement ». Il affirme que cette allégeance à la tradition " conditionne et limite [...] certaines formes d'innovation », mais que " les pratiques d'enseignement offrent également une base pour la consolidation et l'orientation des assertions théoriques ». A. Lunsford répond par un avertissement, affirmant que la tradition a exclu de nombreuses personnes de l'éducation rhétorique, et elle exprime l'espoir que ce domaine si sophistiqué devienne moins exclusif.

Graff, Richard \& Michael Leff. 2005. "Revisionist Historiography and Rhetorical Tradition(s)» [«L'historiographie révisionniste et la/les tradition/s rhétorique/s »], Graff, R., A. Walzer \& J. Atwill (éds), The Viability of Rhetorical Tradition [La viabilité de la tradition rhétorique] (Albany: SUNY Press), 11-30

Graff et Leff affirment que le fait de définir la tradition rhétorique comme une tradition d'enseignement permet à la fois une cohérence de la discipline et une flexibilité au regard des textes, nécessaires au vue de l'élargissement des conceptions de la rhétorique.

Kahl, Mary L. \& Michael Leff. 2006. "The Rhetoric of War and Remembrance: An Analysis of President Bill Clinton's D-Day Discourses » [«La rhétorique de la guerre et du souvenir: une analyse des discours sur le jour J du président Bill Clinton »] Qualitative Research in Communication 7, 15-22

Kahl et Leff analysent quatre des discours de Bill Clinton commémorant le jour J du Débarquement. Ils affirment que Clinton doit négocier son identité en tant que membre de la jeune génération qui n'a pas participé au Débarquement et qui a protesté contre la guerre au Vietnam, et en tant que président qui ne dispose que d'un tiède soutien de la part de l'armée. Ce faisant, il incarne une forme d'argument d'utilité (un raisonnement basé sur le bénéfice attendu) qui "guide la pensée et l'action dans des contextes où les principes abstraits sont insuffisants pour déterminer la conduite ».

Leff, Michael. 2006. «Rhetoric, Dialectic, and the Functions of Argument », Houtlosser, P. \& A. van Rees (eds). Considering Pragma-Dialectics. [ Rhétorique, dialectique et les fonctions de l'argumentation ", Houtlosser, P. \& A. van Rees (éds). Considérer la pragmadialectique] (Mahwah, NJ: Erlebaum), 199-210

Leff plaide en faveur d'une réhabilitation de la rhétorique de la plaidoirie, en complément de la «dialectique rhétorique » de van Eemeren et Houtlosser, et la 
«rhétorique dialectique » de Tindale, basée sur Perelman. Ces conceptions incorporent la rhétorique à la dialectique ou l'inverse. Soutenant que Le Pro Murena de Cicéron «joue une fonction argumentative légitime bien qu'il viole les règles de la pertinence fondées sur des bases dialectiques, Leff avance que la plaidoirie agonique «peut avoir une fonction constructive quand les parties directement impliquées possèdent des opinions solides et fermement ancrées ou sont opposés par des conflits d'intérêts ». Leff, Michael. 2006. «Introduction to Forum: Rhetoric and Society in the $21^{\text {st }}$ Century » [«Introduction au forum: rhétorique et société au $21^{\mathrm{e}}$ siècle »], Quarterly Journal of Speech, 92 51-52

Introduction à un numéro consacré au Colloque de l'Alliance pour les Etudes rhétoriques en 2003, pour lequel les participants ont écrit des exposés sur l'agentivité, la tradition rhétorique, les objectifs institutionnels et sociaux des études universitaires de rhétorique et le sens de l'enseignement de la rhétorique, la composition ou l'art d'exposer.

Leff, Michael. 2006. «Up From Theory: Or, I Fought the Topoi and the Topoi Won » ["Se remettre de la théorie : ou j'ai combattu les Topoi, et les Topoi ont gagné »], Rhetoric Society Quarterly 36, 203-211

Leff raconte l'expérience personnelle de sa première étude des topoï classiques, dans le cadre de laquelle il cherchait une "théorie substantielle de la rhétorique», et comment, en comparant Boèce avec les premiers auteurs latins comme Cicéron et Quintilien, il conclut que les topoï ne possèdent pas une haute valeur théorique. Le but de ce récit est de souligner le changement intervenu dans les études rhétoriques qui sont passées d'un intérêt pour la théorie à un souci des développements politiques et de la pédagogie. Leff fait l'hypothèse que ce changement va provoquer une révision des cursus qui pourrait même aller jusqu'à effacer les frontières des départements.

Leff, Michael. 2008. "Ad Hominem Argument in the Bush/Kerry Presidential Debates ", van Eemeren, Frans \& al. Proceedings of the $6^{\text {th }}$ Conference of the International Society for the Study of Argumentation ["L'argument Ad Hominem dans les débats présidentiels Bush/ Kerry ", van Eemeren, Frans \& al. Actes de la $6^{e}$ Conférence de la Société internationale pour l'étude de l'argumentation] (Amsterdam: SicSat) 859-866 ${ }^{9}$

Leff soutient que, dans les débats présidentiels de 2004, le président George W. Bush utilise l'argument ad hominem plus efficacement que le sénateur Kerry, de manière à la fois valide et plus fallacieuse.

Leff, Michael. 2008. "Introduction: David Zarefsky and Reasonable Argument» [« Introduction: David Zarefsky et l'argument raisonnable »], Argumentation \& Advocacy $45,61-63$

Introduction à un numéro dédié au travail en argumentation du chercheur David Zarefsky.

Leff, Michael. 2008. "Grover Cleveland and the Non-rhetorical Presidency », Medhurst, Martin (ed). Before the Rhetorical Presidency [ "Grover Cleveland et la présidence non rhétorique ", Medhurst, Martin (éd.). Avant la présidence rhétorique] (College Station, TX: Texas A\&M Press), 289-306

Leff soutient que l'apparente aversion du président Cleveland envers l'éloquence provient de la combinaison de la personnalité de celui-ci et de ses valeurs victoriennes individuelles. En raison de ces dernières, la rhétorique des mouvements populistes et 
travaillistes lui était incompréhensible, et il choisit par conséquent de ne même pas en prendre connaissance.

Leff, Michael. 2009. "Perelman, ad Hominem Argument, and Rhetorical Ethos", Argumentation, 23, 301-311, Version française: 2009. «Perelman, argument ad hominem et ethos rhétorique ", Argumentation et Analyse du Discours $2^{10}$

Utilisant l'essai de Dubois « M. Booker T. Washington et d'autres » comme une étude de cas, Leff soutient qu'il existe une "tension fondamentale " à l'intérieur du projet de Perelman. D'un côté, il adopte une perspective entièrement rhétorique sur l'argumentation en insistant sur le traitement des arguments en situation, mais de l'autre, son analyse de l'argumentation découle d'un système dont l'orientation est diamétralement opposée et qui vise à identifier et à classifier des techniques argumentatives abstraites et décontextualisées.

70 Leff, Michael. 2009. "The Expedient, the Honorable, and the Sacred: Rhetorical Topoi and the Religious Imperative ", Pernot, Laurent (ed.). New Chapters in the History of Rhetoric ["L'opportun, l'honorable et le sacré: les topoï rhétoriques et l'impératif religieux ", Pernot, Laurent (éd.). Nouveaux chapitres dans l'histoire de la rhétorique] (Boston: Brill), 401-416

Leff compare "l'oraison funèbre» de Périclès telle qu'elle a été rapportée par Thucydide au «Second discours inaugural » de Lincoln, pour entamer une discussion sur la manière dont le discours religieux complexifie la rhétorique. Il conclut que le langage de la religion civile dans le discours de Lincoln tend à allier l'honorable et l'opportun, alors que le discours nationaliste laïc de Périclès préserve la distinction entre l'honorable et le sacré.

\section{Comptes rendus d'ouvrages}

71 Dorey, T. A. (ed.). 1965. Cicero [Cicéron] (London: Routledge and Kegan Paul), Western speech $31,59-60$

Leff fait la critique les chapitres de R. G. M. Nisbet dans lesquels celui-ci «soutient la position de Platon selon laquelle la rhétorique devrait être appréhendée au moyen de normes morales constantes " et conclut que Cicéron "échoue à maitriser la force morale d'un Démosthène ou d'un Lincoln parce que ses talents étaient trop fréquemment employés en faveur d'une mauvaise cause ». Leff conclut que l'auteur « se trompe en mettant plus l'accent sur des valeurs abstraites et littéraires plutôt que sur des considérations rhétoriques et politiques ».

Landry, Albert M. (ed.(. 1970. Arts Liberaux et Philosophie au Moyen Age. (Montreal: Institut d'Etudes médiévales), Quarterly Journal of Speech 56, 448-449

Leff suggère que ce travail qui «fournit un historique sommaire des arts libéraux au moyen-âge » possède une valeur pour tous ceux qui s'intéressent à l'histoire de la théorie rhétorique parce que ses articles "tentent de découvrir la place que les penseurs médiévaux attribuent à la communication dans le contexte plus large de l'activité philosophique». Leff montre comment les différents penseurs médiévaux considèrent la rhétorique comme épistémique, comme une branche de l'éthique, ou comme faisant partie de la logique. Il conclut que le livre «nous rappelle encore que les problèmes de la nouvelle rhétorique ne sont pas très éloignés de ceux de l'ancienne ». 


\section{nouveaux politiciens dans l'Athènes du quinzième siècle »], Quarterly Journal of Speech} $58,484-485$

Leff avance que l'auteur combine une approche philologique de l'étude textuelle et une approche sociologique de la dynamique de groupes d'une manière qui lui permette de reconstruire un environnement politique ancien. Il suggère que ce travail pourrait être un excellent modèle d'étude hybride intégrant des méthodes des études de lettres et des sciences sociales.

Murphy, James J. 1975. "Rhetoric in the Middle Ages " ["La rhétorique au MoyenAge »] Quarterly Journal of Speech 58, 336-340

Leff suggère que l'auteur a découvert un principe unificateur qui lui permet de définir plus clairement la portée de la théorie rhétorique médiévale. Selon ce principe, les traités médiévaux sur la théorie rhétorique prétendent généralement fournir «l'art de » mettre en pratique l'éloquence dans le cadre d'une certaine forme de composition, par ex. l'écriture épistolaire ou le prédicat. Cependant, il affirme que la manière dont Murphy caractérise la tradition classique, son analyse d'Alcuin, et son interprétation du sort du mouvement épistolaire en Italie sont problématiques.

Gruin, Erich S. 1976. "Last Generation of the Roman Republic " [ "La dernière génération de la République romaine »]. Quarterly Journal of Speech 62, 209-210

Leff note que Gruin appréhende l'histoire politique d'une manière instrumentale en argumentant contre les théories existantes et en utilisant la logique résiduelle pour proposer une nouvelle approche. Il évite de commenter l'exactitude historique des conclusions de Gruin, suggérant néanmoins cette approche de l'histoire politique pourrait être utile aux étudiants en communication politique.

«The Institutiones Oratoriae of Sulpitius Victor». Quarterly Journal of Speech 63 (1977), 441-443

Leff note que Sulpitius substitue l'idée de compréhension, ou intellectio, à celle de mémoire dans la division en cinq parties de la rhétorique. Il soutient que ce changement correspond à un abandon de la présentation orale dans les cours de justice $\mathrm{du}$ haut empire romain. Il s'agit d'une adaptation des traditions classiques plus que d'une rupture nette avec elles.

Lutz, Cora. 1978. «Schoolmasters of the Tenth Century " [«Les maitres d'école au dixième siècle »], Quarterly Journal of Speech 64, 231-232

Leff suggère que ce travail hautement biographique et historique est utile pour les historiens de la rhétorique parce qu'il ajoute du poids aux arguments récents selon lesquels l'intérêt pour la rhétorique a persisté au moyen-âge.

Murdoch, Iris. 1979. «The Fire and the Sun: Why Plato Banished the Artists » [« Le feu et le soleil : pourquoi Platon a-t-il bani les artistes »], Philosophy and Rhetoric 12, 203-205

Leff suggère que ce livre, dans lequel Murdoch tente de réconcilier l'art avec un platonisme modernisé, «suggère une philosophie de la communication beaucoup plus profonde que celle que nous avons pu extraire de lectures isolées de Gorgias et Phèdre ». Il le fait, explique Leff, à travers une interprétation de Timée utilisant le concept de nécessité.

Edens, Kathy. 1998. "Hermeneutics and the Rhetorical Tradition: Chapters in the Ancient Legacy and its Humanist Reception» [«L'herméneutique et la tradition 
rhétorique : Aspects de la tradition antique et de sa réception chez les Humanistes " ], Quarterly Journal of Speech 84, 241-243

Leff suggère que Kathy Eden " a découvert un point d'interconnexion explicite entre la rhétorique et l'interprétation des textes classiques, et qu'elle montre de manière stimulante comment cette interconnexion stimule la théorie et la pratique herméneutiques ». Le seul reproche de Leff, qu'il admet lui-même n'être pas juste en raison des contraintes spatiales, est qu'elle n'illustre pas cette interconnexion par sa propre écriture.

\section{Bibliographies}

Leff, Michael. 1972. "A Basic Library for the Study of Classical Rhetoric ", Murphy, James J. (ed). A Synoptic History of Classical Rhetoric [« Bibliothèque de base pour l'étude de la rhétorique classique ", Murphy, James J. (éd.). Histoire synoptique de la rhétorique classique] (New York: Random House), 188-192

1 Leff, Michael. 1974. "A Selected Bibliography of Rhetorical Studies for the Year 1973 ", Patrick Kennicott (ed.). Bibliographic Annual in Speech Communication [«Bibliographie sélective des études rhétoriques pour l'année 1973 ", Patrick Kennicott (éd.). Bibliographie annuelle de Communication verbale] (New York: SCA), 75-89

22 Leff, Michael. 1975. "A Selected Bibliography of Rhetorical Studies for the Year 1974 ", Kennicott, Patrick (ed.). Bibliographic Annual in Speech Communication ["Bibliographie sélective des études de rhétorique pour l'année 1974 », Kennicott, Patrick (éd.). Bibliographie annuelle de Communication verbale] (New York: SCA), 45-61

83 Leff, Michael \& Steven R. Edscorn. 2009. «Bibliographie sélective annotée des publications en langue anglais sur la rhétorique, la critique rhétorique et l'argumentation ", Argumentation et Analyse du Discours 2

\section{NOTES}

1. En référence à la pièce d'Aristophane Les oiseaux dans laquelle deux habitants d'Athènes décident d'ériger une ville parfaite dans les nuages, nommée Coucou-les-Nuées (Note de la traductrice).
2. Article disponible dans ce numéro.
3. Article disponible dans ce numéro.
4. Adaptation du style au sujet (note de la traductrice)
5. Voir note 4.
6. Article disponible en français.
7. Partisan de Hicks, prédicateur Quaker de la fin du 18e siècle, abolitionniste et libre-penseur.
8. Article disponible dans ce numéro.
9. Article disponible dans ce numéro.
10. Article disponible en Français. 


\section{AUTEURS}

\section{STEVEN R. EDSCORN}

Memphis Theological Seminary 